\title{
CONTRIBUTIONS TO THE KNOWLEDGE OF BULBAR AUTONOMIC CENTRES
}

\section{VESICO-RECTAL RESPONSES TO ELECTRICAL STIMULATION OF THE MEDULLA OBLONGATA}

\author{
MASARU KURU* AND GESSEN HUKAYA $\dagger$ \\ Neurological Laboratory of Surgical Clinic, Kanazawa University \\ School of Medicine, Kanazawa
}

In a previous paper one of us (M.K.) has pointed out the existence of 2 kinds of centripetal connections between the inferior part of the spinal cord and the medulla oblongata, the site of termination of the both being closely adjacent to each other. The one which ascends in the superficial layer of the paramedian portion of the posterior funiculus, is composed of protoneurons, the nerve-cells of which exist in the posterior-root ganglia, sending their peripheral branches to pelvic organs. The other, which ascends in the lateral funiculus, is composed of deuteroneurons, the nerve-cells of which seem to exist in the intermediate zone of the spinal grey mass, where they are thought to be connected with axons of peripheral neurons. In another paper he, with Yamamoto and Sugihara, elucidated the relation of the former to visceral sensations by recording from the paramedian superficial fibres of the posterior funiculus the action potential brought about by filling the urinary bladder. This finding as well as the scrupulous investigations of Barrington concerning the reflex contraction of the bladder (see later) suggest the existence of viscero-motor centres within the medulla oblongata for the pelvic organs too. The experiments to be described were made with a view of localizing the part of the brain with which reflexes for vesico-rectal contraction and relaxation are connected.

\section{METHODS}

Dogs were used. Under intraabdominal administration of $0.05 \mathrm{~g}$ allobarbital or isomytal per kilo body weight, the carotid arteries were ligated on both sides and successively artificial respiration was commenced with the insertion of a tracheal cannula. With suboccipital craniotomy and removal of the cerebellum, the floor of the fourth ventricle was exposed. Throughout the intracranial manipulation great care was taken of avoiding haemorrhage and the slightest injury to the bulb. For the stimulation a unipolar electrode with a tip of 0.08 $\mathrm{mm}$. in diameter was used. This subserved the cathode, the anode being fixed to the subcutaneous tissue inside the operation wound. Now, inserting the electrode at random at various points of the floor of the fourth ventricle in

Received for publication March 7, 1954.

* 久留 勝 Now Chief of the 2nd Surgical Clinic, Osaka University School of Medicine.

十梁谷月泉 
varying depths, electrical stimulation of $0.1 \mu \mathrm{F}$ condenser discharge was applied under thyratron control, voltage being $0.8 \mathrm{~V}$ and frequency 50 per sec. Changes in the tone of the bladder and rectum, which occurred, were recorded simultaneously through a catheter (for the former) and a rubber balloon (for the latter) connected to Marey's tambour upon smoked paper. After each stimulation, regardless of the occurrence of vesico-rectal responses, the site of the electrode's tip was marked by a $3 \mathrm{sec}$. discharge of $0.1 \mathrm{~mA}$ direct current. The development of hydrogen gas destroys the spot where the electrode's tip was situated. The spots were then traced precisely in serial sections with Weigert stain.

RESULS

In 25 dogs about 500 points were stimulated, about one fifth of these stimulations elicited responses recorded as changes in the pressure in the bladder or the rectum or, more frequently, in both of them. In general, each successful stimulation was followed either by a single contraction or by a relaxation with consecutive contraction; or rarely by relaxation with an initial contraction.

With precise scrutiny of the site of each responded point, it is not difficult to point out certain anatomical characteristics in regard to the distribution of points with different responses.

The part of the bulb, stimulation of which evokes contraction of the bladder or rectum (fig. 1), is located between the level of the most rostral end of the ala cinerea and the pyramidal decussation; and the strongest contraction can be elicited by stimulation of the grey mass encircling the solitary fascicle, especially at the level directly caudal to the obex (fig. $3 \mathrm{D}$ ). In no single case was the response obtained by stimulation of the parts above the ala cinerea in our experiments. Rostrally to the obex, the points where responses were elicited, were concentrated in the grey mass closely adjacent to the solitary fascicle running parallel to the extent of the ala cinerea (fig. 3 and 4). Caudad to the obex, these bilateral grey masses surrounding the solitary fascicle tend to fuse into one. Conforming to this anatomical arrangement, the effective points also tend to flock together there. At this level stimulation of the grey mass surrounding the solitary fascicle can be more easily done, and reactions observed were usually more evident than in the stimulations applied more caudally or rostrally. The points of effective response become to locate progressively afar from the central canal as it goes more caudally from the level of the obex, where the lemniscal and pyramidal decussations gradually develop. At the most caudal level of the pyramidal decussation they are observed in the internal half of the anterior horn and in its vicinity. In no case was a response recorded subsequent to stimulations given caudal to the pyramidal decussation.

The part of the bulb, stimulation of which resulted in a marked relaxation of the two pelvic organs (fig. 2), was located between the most rostral end of the ala cinerea and the level directly caudal to the obex. The points of response seemed to be concentrated especially in the reticular formation ventral or ventrolateral to the solitary fascicle (fig. 3 and 4 ). Similar to the case of contraction, 

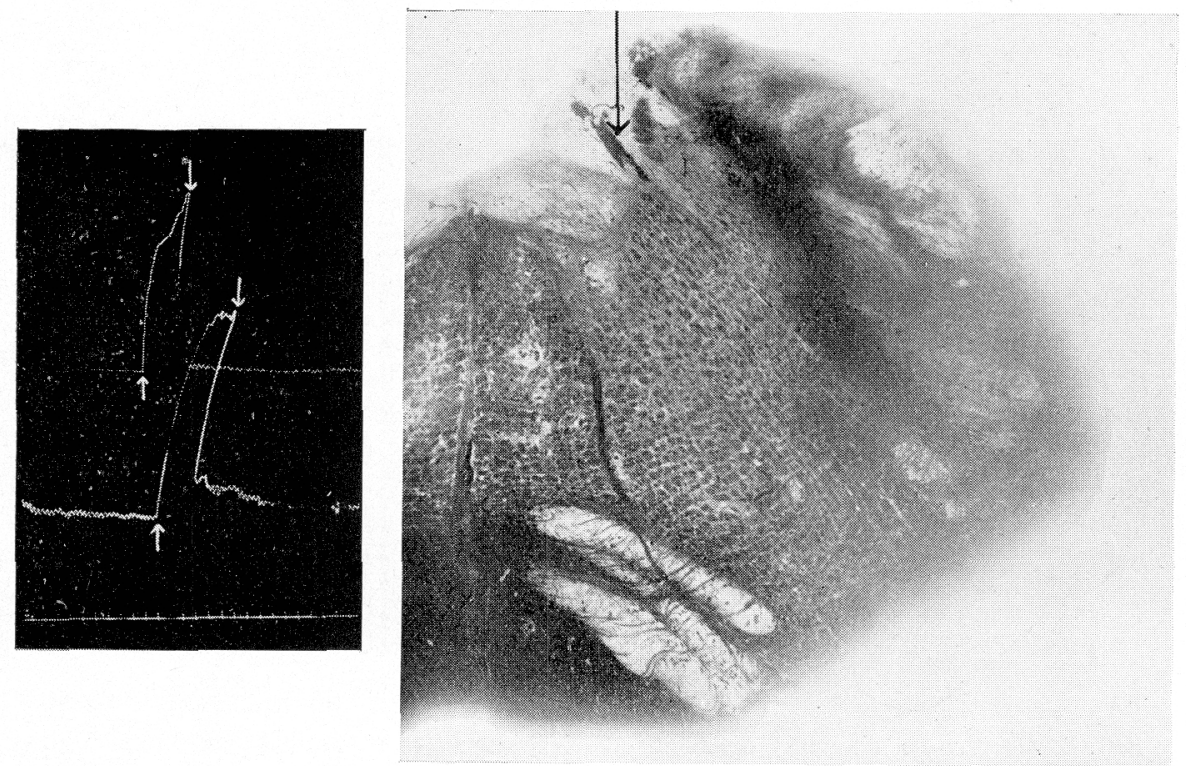

FIG. 1. Remarkable contractions of the rectum and bladder elicited by stimulation of the part closely adjacent to the solitary fascicle at the level a little rostral to the obex.

Right figure: Stimulated point is marked with an arrow.

Left figure: Rise in the tone of the rectum (upper) and the bladder (lower). Duration of stimulation is marked with arrows. Time in 6 sec.
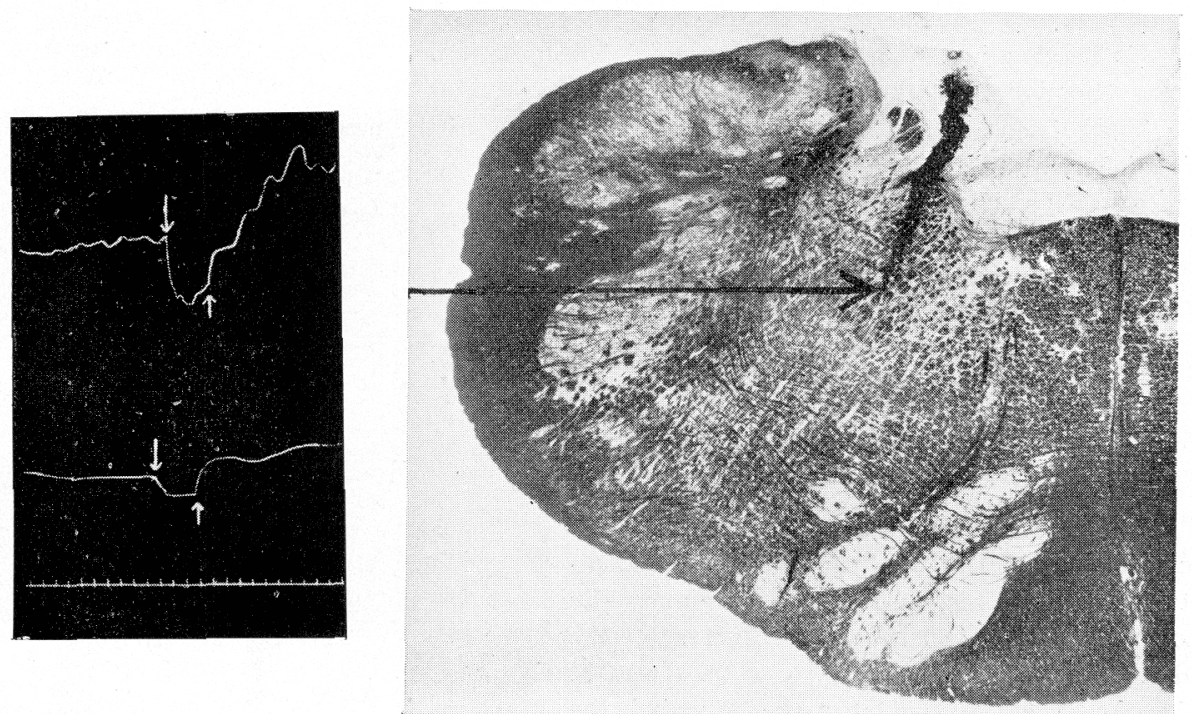

FIG. 2. Relaxations of the rectum and bladder elicited by stimulation of the part of reticular formation ventral to the solitary fascicle and medial to the spinal root of the trigeminus at the level of middle of ala cinerea.

Right figure: Stimulated point is marked with an arrow.

Left figure: Fall in the tone (with successive rise) of the rectum (upper) and the bladder (lower). Duration of stimulation is marked with arrows. Time in 6 sec. 

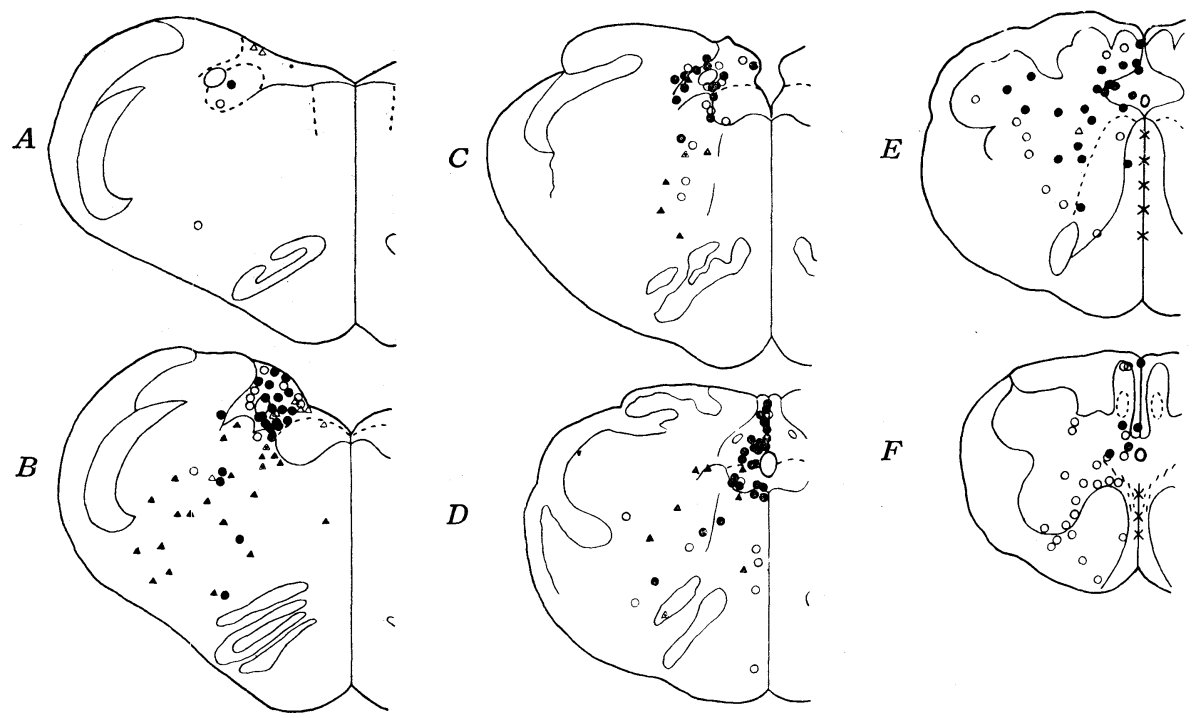

FIG. 3. Diagrams showing distribution of points of recto-vesical responses following bulbar stimulation. Results obtained in $25 \mathrm{dogs}$ on both side of the medulla were transferred to the right half superimposing points with responses on neighbouring sections upon respective representative section.

$A$ : Section through the rostral part of ala cinerea.

$B$ : Section through the middle of ala cinerea.

$C$ : Section through the caudal part of ala cinerea.

$D$ : Section just caudal to the obex.

$E$ : Section just rostral to the pyramidal decussation.

$F$ : Section through the caudal part of the pyramidal decussation.

The relation of these sections to surface anatomy of the medulla is shown on the right side of fig. 4.

- Conspicuous contraction.

- Slight contraction.

- Conspicuous relaxation.

$\triangle$ Slight relaxation.

$\triangle$ Contraction with successive relaxation.

FIG. 4. Dorsal view of the lower brain stem of the dog with the cerebellum removed. The location of points of constrictory (left) and dilatatory (right) responses is diagrammatically shown projected upon the dorsal surface of the bulb. The letterling from $A$ to $F$ on the right side of the figure indicates the level of the sections shown in fig. 3 .

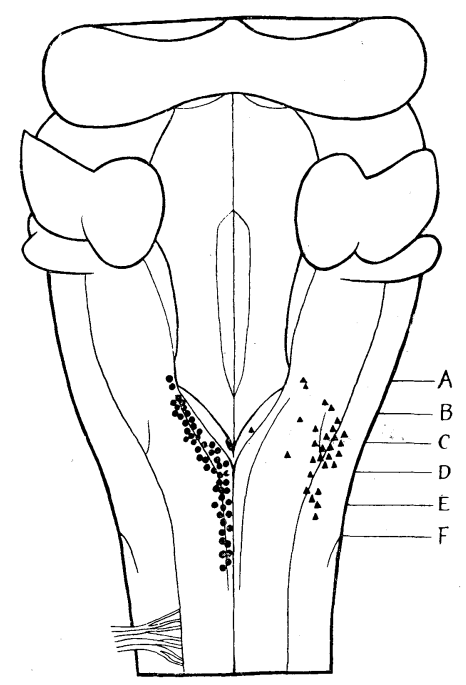


response could not be recorded when the parts rostral to the ala cinerea were stimulated. Points of relaxative response were found especially abundantly in the reticular substance near the basal grey mass of the part caudal to the rostral end of the ala cinerea. In this part of the reticular substance they were considerably dispersed, the most ventral one of them being situated near the dorsal border of the inferior olive and the most dorsal one near the ventral margin of ala cinerea (fig. $3 \mathrm{~B}$ ). At the level of the middle of the ala cinerea they show tendency of concentrating in the medial half of the reticular substance ventral to the hypoglossal nucleus. Stimulations of the lateral half of the reticular formation remained ineffective, a circumstance which could also be confirmed at the level directly caudal to the obex. Stimulation of any parts caudal to this level hardly elicited vesico-rectal response.

It is noteworthy that in all cases where relaxation was recorded, a successive contraction of considerable duration followed (fig. 2). The contraction, however, was not constantly followed by relaxation. While the bilateral areas of the points causing contraction gradually converge towards the caudal direction so that they fuse in the part of medulla directly caudal to the obex, those of the points causing relaxation are situated rather in the rostral half of medulla and entirely separated on both sides. This fact may be the reason, why relaxation is more difficult to elicit than contraction. It seems also likely that the former points are aggregated in the form of a dense cylinder, while the latter are dispersed in the form of a porous sponge.

It was related above that in a few cases a stimulation caused an initial slight contraction which was followed by relaxation. This was a phenomenon observed in stimulation of the reticular substance just ventral to the basal grey matter of the fourth ventricle, where in most experiments relaxation only was noticed. The reason why the stimulation of the similar part of the bulb evokes different response in different experiments is not yet perfectly elucidated.

SHORT ACCOUNTS OF LITERATURE

Budge $(1858,1864,1872)$ stimulated electrically various parts of the central nervous system from the cerbral peduncle to the sacral region of the cord, and, recognizing contraction of the bladder subsequent to stimulation of all the parts except for the cerebellum, came to the conclusion that the vesical centre in the brain stem exists in the cerebral peduncle. Karplus and Kreidl (1909) could observe constantly vesical contraction by electrical stimulation of the base of the hypothalamus between the optic and oculomotor nerves.

With scrupulous experiments on cats Barrington $(1921,1925)$ pointed out the existence of 5 kinds of reflex concerning micturition. Among others the centres for the two important reflexes in which powerful and sustained contractions of the bladder appeared were found to be situated not in the spinal cord, but in the brain stem. In further investigations he located their sites in the part of the brain stem just above the rostral top of the medulla.

Ranson, Kabat and Magoun, utilizing Clarke-Horsley's stereotaxic instrument, stimulated the hypothalamus, preoptic region and septum and confirmed that the vesical contraction was resulted by stimulation of the grey masses 
adjacent to the anterior commissure and the septum while the inhibition of the intestinal peristalsis and the contraction of the urinary bladder by stimulation of the hypothalamus. Wang and Ranson in their study of the autonomic response to the electrical stimulation of the brain stem alluded to the reaction in the bladder and illustrated the relation in diagrams. Their conclusions will be referred to later. Lichtenstern analysed the vesical centre in diencephalon by means of electrical stimulation. Takeda also mentioned vesico-rectal responses in his study on the sympathetic and parasympathetic centres within the hypothalamus.

Kusunoki and his collaborators ascertained the inhibitory effect of picrotoxine upon the tone of the stomach, small intestine, descending colon, rectum and urinary bladder, when injected intravenously in rabbits. Dividing the brain stem at various levels, they came to the conclusion that the drug affects the sympathetic centres in the brain stem. The centre for the tone of the descending colon was found to be located in the caudal portion of the nuclei pontis.

Relatively few investigations are published concerning the bulbar control of the pelvic organs beside those of Budge and Wang and Ranson above referred to.

Hatcher and Weiss recognized the vomiting centre to be located in the dorsal sensory nucleus of the vagus nerve in the medulla. They also noticed that defecation was resulted by stimulating its neighbouring parts, the fact which was affirmed by Kappanyi in experiments in quest of the bulbar defecation centre performed with a similar method. Since the process of defecation, similar to that of vomiting, is a complex mechanism involving various elements, it is not prudent to relate their results directly with ours. Nevertheless, a certain coincidence between the two cannot be overlooked.

Chen, Lim, Wang and Yi have performed a series of experiments to prove the bulbar sympathetic centres. Among various kinds of responses to the electrical stimulation of the medulla they recorded reactions in the bladder and descending colon. As it is almost sure that those organs are independent of the vagal innervation, their description should be considered in detail. In their first paper they recorded relaxation of the bladder and descending colon subsequent to stimulation of the pressor point which was described by Ranson and Billingsley, besides various sympathetic effects in other organs. In their third paper, they referred to the fact that in the medulla points causing the maximal responses in different organs do not coincide with each other but are dispersed upon the inferior half of the floor of the fourth ventricle. Concerning the responses in the bladder and descending colon, their experiments were performed upon dogs thus permitting comparison with ours. It is to be regretted, however, that in their description, the points of stimulation were only superficially projected upon the floor of the fourth ventricle, without considering the depth to which the electrode's tip was inserted. If attention was paid to depth, they might have reached another conclusion. In this paper eleven points upon the floor of the fourth ventricle, stimulation of which resulted in contraction in the above two organs, were presented in a diagram. It is worthy of note that most of these points are situated close to the ala cinerea, as a similar condition 
is also seen in our experiments. Unfortunately in this diagram, the points for provoking relaxation of the bladder as reported in their first paper, were not drawn.

Wang and Ranson, utilizing bipolar electrodes connected to Clarke-Horsley's stereotaxic instrument, stimulated various parts of the brain stem from the mesencephalon to the inferior part of medulla and recorded responses in the blood-pressure, urinary bladder and iris, and presented the distribution of the effective points in clear diagrams. According to them the vesical response can be elicited, not only by medullar stimulation, but also by pontine and mesencephalic stimulations. Even in the medullar stimulation, the points causing vesical response are considerably dispersed, though they show a certain tendency to be located more densely in the ventro-lateral half of the reticular substance. Similar investigations was performed by Monnier too. According to him, the electrical stimulation of the ventro-lateral part of the bulbar reticular formation and the area adjacent to the rephe was resulted in contraction of the bladder.

\section{DISCUSSION}

It should be emphasized that in our experiments a unipolar electrode with a fairly fine tip $(0.08 \mathrm{~mm}$.) was employed and accordingly that the strength of electrical stimulation was much weaker than that in the experiments of previous investigators. It is generally assumed, that the unipolar electrode is less reliable in the restriction of the stimulating area than the bipolar one. This disadvantage can be readily overcome by diminuition of the strength of stimulus. Since in our experiments, as stated above, the two opposite responses, contraction and relaxation, could be recorded separately by stimulations applied to two points adjacent each other within a distance of $1 \mathrm{~mm}$., it may safely be said that a stimulus acts restrictedly a sphere of less than $1 \mathrm{~mm}$. in diameter. Moreover, with use of the unipolar electrode injury of the medullary substance can be restricted to less than half that necessarily encountered in the use of the bipolar electrode.

It is almost impossible to make uniform the depth of narcosis in each experiment, especially in dogs as their size varies considerably from one another. And insufficient narcosis makes performance of experiments impossible, while excess in it results in abolition of the reactions in quest. For this kind of experiment the administration of allobarbital or isomytal is most convenient. In a certain number of dogs, experiments were made on decebrate state. However, in half the decerebrate animals artificial respiration was necessary and even with that some of them succumbed to haemorrhage within a few hours. But taking into consideration the results of experiments in such animals with artificial respiration, we endeavoured to distinguish the influence of respiration upon the vesico-rectal reaction. It can easily be discerned whether the reaction in the bladder and rectum is deformed by respiration or not. It is, however, necessary to keep in mind that the results above stated were deduced from experiments in decerebellate animals under the influence of narcotics, such as barbiturate derivates. 
Among the experiments mentioned above, the conditions of the experiments by Wang and Ranson, Monnier, and Chen and his collaborators were similar to ours. But the conclusion of Wang and Ranson differed considerably from ours in the results of stimulations applied to parts beyond the rostral end of the ala cinerea. In their experiments, the descending fibres from the hypothalamic region were likely stimulated together as stronger stimulations were used by them. In conformity with us, however, they have also noticed that, in some cases, a single stimulation caused a contraction followed by a relaxation of the bladder. The results of Monnier were almost opposite to ours, since he recorded vesical contraction by stimulating the ventro-lateral portion of the bulbar reticular formation. It will, however, be noted that the vesical contraction in his experiments seems to have followed not immediately the stimulation, as is the case in our experiments, but with certain latency. The results of Chen et al. is very important in having ascertained that electrical stimulation of the medulla evokes manifest reactions in the bladder and descending colon, though their experiments seem to have been made chiefly on the pressor area of Ranson and Billingsley with stimulated points superficially projected upon the floor of the fourth ventricle.

In comparison with such findings of previous investigators, the following two points may be mentioned as characteristics of our experimental results: First, the area in the medulla which has been found as causing vesico-rectal responses is far more restricted than the areas indicated by others. Second, the area for causing contraction can be discriminated to some extent from that causing relaxation.

Though it should not be rashly decided whether those reactions are evoked by stimulation of the nerve-cells or of the nerve fibres connected to them, it does not seem unnatural to locate in these areas of the medulla, the bulbar autonomic centres for these pelvic organs, for similar stimuli, if applied to the part of the pons just rostral to the rostral end of the bulb or to the part of the spinal cord just caudal to the bulb, proved to be ineffective.

This conclusion is supported by the facts that the site of termination of the sacro-bulbar connections, as above stated, is closely related to the above centres; that one of these sacro-bulbar connections is proved to mediate the sense of distension of the urinary bladder; and that the reflex of micturition is mostly dependent upon the existence of the part of brain stem, which, as Barrington elucidated clearly, is situated between the caudal level of the inferior colliculi and the pyramidal decussation.

In other words, important autonomic centres for defecation and micturition exist in the medulla and the origins of centrifugal fibres for these reflexes are situated in grey masses near the solitary fascicle closely adjacent to the dorsal nuleus of the vagus nerve and in the reticular formation ventral to it. It can be traced that the ascending fibres from the inferior level of the spinal cord terminate at these grey masses.

As to the connections of these centres with higher centres, we refer to the experimental works of Barrington, Wang and Ranson and Kurotsu and as to those to the spinal centres, the works of Chen and his collaborators. In our 
laboratory, too, experiments concerning this respect are in progress which will be published in near future.

\section{CONCLUSION}

On narcotized, carotid-ligated, and decerebellate dogs, a thin electrode was inserted through the floor of the fourth ventricle. Various parts of the medulla were stimulated by $0.1 \mu \mathrm{F}$ condenser discharge (voltage $0.8 \mathrm{~V}$, freqeuncy 50 per sec., thyratron control) and reactions in the bladder and rectum were recorded. The points of tissue where stimulations were proved effective were determined histologically in serial sections.

Subsequent to the stimulation, reactions in the bladder and rectum, similar in type, usually appeared simultaneously; exceptionally, however, one of these two only responded with the other remaining quiescent.

The points, where stimulation caused contraction of the bladder or rectum are distributed between the rostral top of the ala cinerea and the caudal end of the pyramidal decussation (fig. 3 and 4). Especially the stimulation of the grey matter neighbouring the solitary fascicle at the level directly caudal to the obex evoked constantly conspicuous contraction of both organs.

The distribution of the points, stimulation of which evokes relaxation of these organs, is confined to an area between the most rostral portion of the ala cinerea and the level just caudal to the obex. It is especially dense in the reticular formation ventral or ventro-lateral to the solitary fascicle at the middle of ala cinerea (fig. 3 and 4 ).

Since the terminations of the sacro-bulbar connections, as previously elucidated by one of us, are closely adjacent to them, and since evidence was presented that by one of those connections (pelvic sensory vagus) the sense of distension of the urinary bladder is mediated, we can conclude that in the medulla oblongata the autonomic centres exist, which are presumably most important for the reflex of micturition and defecation, and that they are situated close to those of the thoraco-abdominal viscera.

The expences for this work were defrayed by a grant for the scientific research from the Department of Education.

\section{REFERENCES}

1. BARRINGTON, F. J. F. The relation of the hind brain to micturition. Brain $44: 23-32$, 1921.

2. BARRINGTON, F. J. F. The effect of lesion of the hind- and midbrain on micturition in the cat. Quart. J. exper. Physiol. $15: 81-102,1925$.

3. BUDGE, J. Über das Centrum genito-spinale des N. sympathicus. Virchow's Arch. $15: 115-126,1858$.

4. Budge, J. Über den Einfluss des Nervenensystems auf die Bewegung der Blase. $Z$. rat. $M e d$. 3. Reihe, 21:1-16, 174-191, 1864.

5. Budge, J. Zur Physiologie des Blasenschliessmuskels. Pflüger's Arch. 6: 306-331, 1872.

6. CHEN, M. P., K. S. LIM, S. C. WANG AND C. L. YI. On the question of a myelencephalic sympathetic centre. I. The effect of stimulation of the pressor area on visceral function. Chin. J. Physiol. $10: 445-482$, 1936. II. Experimental evidence for a reflex 
sympathetic centre in the medulla. ibid. $11: 355-366,1937$. III. Experimental localization of the centre. ibid. $11: 367-384,1937$.

7. HAtcher, R. A. AND S. Weiss. Studies on vomiting. J. Pharm. exper. Therap. 22 : 139-193, 1924.

8. KAPPANYI, TH. Studies on defecation, with special reference to a medullary defecation center. J. labor. a. clin. Med. 16:225-238, 1930.

9. KARPLUS, J. P. AND A. KREIDL. Gehirn und Sympathicus; I. Mitteilung, Zwischenhirnbasis und Halssympathicus. Pflüger's Arch. 129: 138-144, 1909.

10. Kurotsu, T. The highest autonomic centres. (Japanese). Nipponrinsho 8: 971-977, 1950.

11. KURU, M. On the pelvic equivalent of the sensory vagus. Jap. J. Physiol. 1:240-253, 1951.

12. KURU, M., S. YAMAMOTO AND S. SUgiharA. On participation of the fibres of posterior funiculus in the mediation of the visceral sensations from organs in the pelvic cavity. A study on function of the "pelvic vagus." (Preliminary report). Proc. Jap. Acad. 29: 230-233, 1953.

13. KUSUNOKI, G. On the sympathetic viscero-motor centres. (Japanese). Fukuokaigakuzasshi 354 : 281-282, 1942.

14. Lichtenstern, R. Über die zentrale Blaseninnervation. Ein Beitrag zur Physiologie des Zwischenhirns. Wien. klin. Wschr. 25 : 1247-1249, 1912.

15. MonNier, M. Les centres végétatifs bulbaires (effets de l'excitation faradique du bulbe sur la respiration, la tention arterielle, le pouls, la vessie et la pupille chez le chat). Arch. internat. de Physiol. 49: 445-463, 1939.

16. RANSON. S. W. AND P. K. BILLINGSLEY. Vasomotor reactions from stimulation of the fourth ventricle. Amer. J. Physiol. 41:85-90, 1916.

17. RANSON, S. W., H. KABAT, AND H. W. MAgOUn. Autonomic responses to electrical stimulation of hypothalamus, preoptic region and septum. Arch. Neur. 33: 467-477, 1933.

18. TAKEDA, M. On movements of the digestive tube subsequent to electrical stimulations of the autonomic centres. (Japanese). Osakaigakukaizasshi $2: 335-355,1950$.

19. WANG, S. C. AND S. W. RANSON, Autonomic responses to electrical stimulation of the lower brain stem. J. Comp. Neur. 71: 437-455, 1939. 